Ikegami, T.

Osaka J. Math.

14 (1977), 271-289

\title{
ON THE REGULARITY OF BOUNDARY POINTS IN A RESOLUTIVE COMPACTIFICATION OF A HARMONIC SPACE
}

\author{
Teruo IKEGAMI
}

(Received June 6, 1976)

\section{Introduction}

The study of regular boundary points for the Dirichlet problem is one of the most interesting materials in the potential theory. In the case of bounded domains of Euclidean space, various criterions of regularity are given by many authors. Among all, we are interested in the characterization of H. Bauer [1], on account of its extremal character. If we are going to discuss the Dirichlet problem for the whole space, we shall need to introduce the ideal boundary. This is nicely performed when we consider the resolutive compactification of spaces. For the condition of resolutivity of compactification we know it fairly well, but, in contrast with the resolutivity, little is known about the regularity of boundary points. Our present investigations start from the question: does every resolutive compactification contain at least one regular point ? However this is negatively answered by a simple example (Example 1, §3). Hence, we proceed to the problems to characterize the regularity, to give a sufficient condition for the existence of regular boundary points and to study some extremal property of boundary sets. We observe that the lack of exterior points causes difficulties, for in the classical case of bounded domains we know that the exterior of domains plays an essential role.

In the sequel, we shall fix a resolutive compactification of a strict harmonic space $X$ in the sense of Bauer [2]. Hypothesis, definitions and notations used in this paper are stated in $\S 1$. In $\S 2$, a regular boundary point is characterized by its extremal property (Theorem 1). And conditions for the existence of at least one or sufficiently many regular boundary points are given. $\$ 3$ deals with more restrictive regularity, the strong regularity and the pseudo-strong regularity, and the relations among them. It contains also a new sufficient condition for regularity. Relations between the minimal determining sets for some family of hyperharmonic functions, i.e., the Šilov boundary, and the harmonic boundary are established in $\$ 4$. In the last section, we consider open subsets of $X$ and obtain the result that every regular boundary point is strongly regular. 


\section{Preliminaries}

Let $X$ be a strict Bauer space with countable basis, i.e., $X$ satisfies the axioms I, II, III and IV of Bauer [2] and for each point $x$ of $X$ there exists a potential $p$ strictly positive at $x$. We know that $X$ has a finite continuous potential which is strictly positive. We suppose that $X$ is connected and constant functions are harmonic.

Let $X^{*}$ be a compactification of $X$ and $\Delta=X^{*} \backslash X$. Given a numerical function $f$ on $\Delta$, we consider a family of hyperharmonic functions $u$ on $X$, bounded from below and satisfy $\lim _{x \rightarrow y} u(x) \geq f(y)$ for every $y \in \Delta$. The lower envelope of this family is denoted by $\bar{H}_{f}(a)$. We define also $\underline{H}_{f}=-\bar{H}_{(-f)}$. If $\bar{H}_{f}=\underline{H}_{f}$ and are harmonic on $X, f$ is termed to be resolutive and the harmonic function is denoted by $H_{f}$.

A compactification is called resolutive if every $f \in \boldsymbol{C}(\Delta)$ is resolutive, where $\boldsymbol{C}(\Delta)$ denotes the set of all functions finite and continuous on $\Delta$. In the following we shall consider a resolutive compactification $X^{*}$ of $X$.

For a non-negative hyperharmonic function $v$ on $X$ and a subset $E$ of $X$, we define the reduced function

$$
R_{v}^{E}(a)=\inf \left\{u(a) ; \quad \begin{array}{l}
u \text { is a non-negative hyperharmonic } \\
\text { function on } X \text { satisfying } u \geq v \text { on } E
\end{array}\right\} .
$$

The lower semicontinuous regularization

$$
\hat{R}_{v}^{E}(a)=\lim _{a^{\prime} \rightarrow a} R_{v}^{E}\left(a^{\prime}\right)
$$

is hyperharmonic. We have $\hat{R}_{v}^{E}=R_{v}^{E}$ for every open set $E$.

We set

$$
\Gamma=\cap\left\{\Gamma_{p} ; p \text { is a strictly positive potential on } X\right\}
$$

and

$$
\Lambda=\Delta \backslash \Gamma,
$$

where $\Gamma_{p}=\left\{x \in \Delta ; \lim _{a \rightarrow x} p(a)=0\right\} . \quad \Gamma$ is called the harmonic boundary of $X$.

Throughout this paper we shall use the following notations:

$$
\overline{\mathscr{H}}=\{v \text { : superharmonic and bounded from below on } X\} ;
$$

for $v \in \overline{\mathcal{M}}$,

for $x \in \Delta$,

$$
\begin{aligned}
& u_{v}: \text { the greatest harmonic minorant of } v \\
& \left.p_{v}: \text { the potential part of } v \text { (we have } v=u_{v}+p_{v}\right)
\end{aligned}
$$

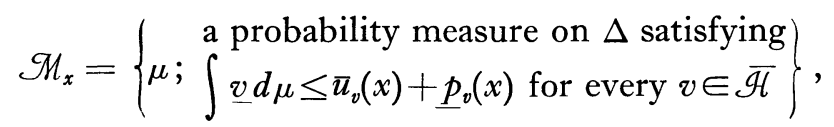


where $\underline{f}$ (resp. $\bar{f}$ ) is the lower (resp. upper) semicontinuous extension of $f$ on $\Delta$. A compact subset $S$ of $\Delta$ is called determining for $\mathscr{F} \subset \overline{\mathcal{H}}$ if

$$
\inf _{S} \underline{v}=\inf _{X^{*}} \underline{v} \quad \text { for all } v \in \mathscr{F} ;
$$

the smallest set determining for $\mathscr{F}$ is called the Silov boundary for $\mathscr{F}$ and is denoted by $S_{\mathscr{F}}$.

Finally, a boundary point $x$ is called regular if

$$
\lim _{a \rightarrow x} H_{f}(a)=f(x) \quad \text { for every } f \in \boldsymbol{C}(\Delta) .
$$

It is known that each point of $\Lambda$ is irregular.

\section{Existence theorem}

As we have mentioned in the introduction, it is not true that every resolutive compactification has a regular boundary point. Thus it becomes an interesting subject to find conditions under which a resolutive compactification contains at least one regular boundary point. For this purpose, we characterize first a regular boundary point by an extremal property, which is a version of that given by $H$. Bauer [1].

Lemma 1. If $x \in \Gamma$ then

$$
\lim _{a \rightarrow x} H_{f}(a) \leq f(x) \leq \varlimsup_{a \rightarrow x} H_{f}(a) \quad \text { for every } f \in \boldsymbol{C}(\Delta) .
$$

Proof. Let $f \in \boldsymbol{C}(\Delta)$. We have a strictly positive potential $p$ such that

$$
\underline{\lim }\left(H_{f}+p\right) \geq f \text { and } \overline{\lim }\left(H_{f}-p\right) \leq f \quad \text { on } \Delta^{1)} .
$$

Then,

$$
\begin{aligned}
\lim _{\bar{a} \rightarrow x} H_{f}(a) & =\lim _{\bar{a} \rightarrow x} H_{f}(a)-\lim _{\bar{a} \rightarrow x} p(a)=\lim _{a \rightarrow x} H_{f}(a)+\varlimsup_{a \rightarrow x}[-p(a)] \\
& \leq \varlimsup_{a \rightarrow x}\left[H_{f}(a)-p(a)\right] \leq f(x) \leq \lim _{a \rightarrow x}\left[H_{f}(a)+p(a)\right] \\
& \leq \varlimsup_{a \rightarrow x} H_{f}(a)+\lim _{a \rightarrow x} p(a)=\varlimsup_{a \rightarrow x} H_{f}(a), \quad \text { q.e.d.. }
\end{aligned}
$$

Thus we have

Corollary 1. A point $x \in \Gamma$ is irregular if and only if there exists $f \in C(\Delta)$ such that

$$
\lim _{\bar{a} \rightarrow x} H_{f}(a)<\varlimsup_{a \rightarrow x} H_{f}(a) .
$$

Lemma 2. If $x$ is regular then $\mathscr{M}_{x}=\left\{\varepsilon_{x}\right\}$.

1) [4] Lemme 3.2.8 and Lemme-clef 2.1.7. 
Proof. Suppose that $\mu \in \mathscr{M}_{x}$ and $\mu \neq \varepsilon_{x}$ then there exist a point $y$, a neighborhood $U(y)$ of $y$, a function $f \in \boldsymbol{C}(\Delta)$ and a strictly positive potential $p$ such that

$$
\left\{\begin{array}{l}
y \in \operatorname{Supp} \mu \backslash\{x\}, \text { where Supp } \mu \text { denotes the support of } \mu \\
x \notin \overline{U(y)}, \mu(\Delta \cap U(y))>0 \\
f \geq 0, f(x)=0 . \quad f>0 \text { on } U(y) \cap \Delta \\
\lim \left(H_{f}+p\right) \geq f \text { on } \Delta .
\end{array}\right.
$$

From (2.1) we derive

$$
\int \lim \left(H_{f}+p\right) d \mu \geq \int f d \mu \geq \int_{U(y) \cap \Delta} f d \mu>0 .
$$

In view of the definition of $\mathscr{M}_{x}$ and $x \in \Gamma$

$$
\int \underline{\lim }\left(H_{f}+p\right) d \mu \leq \varlimsup_{a \rightarrow x} H_{f}(a) .
$$

Since $x$ is regular we arrive at the contradiction

$$
0<\varlimsup_{a \rightarrow x} H_{f}(a)=f(x)=0
$$

which proves the lemma.

Theorem 1. A point $x \in \Gamma$ is regular if and only if $\mathscr{M}_{x}=\left\{\varepsilon_{x}\right\}$.

Proof. It is sufficient to prove that under the condition $x \in \Gamma \mathscr{M}_{x}=\left\{\varepsilon_{x}\right\}$ implies that $x$ is regular. Suppose that $x$ is irregular. Then, by Corollary 1, there exists $f_{0} \in \boldsymbol{C}(\Delta)$ with

$$
\lim _{a \rightarrow x} H_{f_{0}}(a)<\varlimsup_{a \rightarrow x} H_{f_{0}}(a) .
$$

We select a number $\gamma$ such that

$$
\lim _{a \rightarrow x} H_{f_{0}}(a)<\gamma<\varlimsup_{a \rightarrow x} H_{f_{0}}(a) \text { and } \gamma \neq f_{0}(x) .
$$

For each $f \in \boldsymbol{C}(\Delta)$ we define

$$
P(f)=\varlimsup_{a \rightarrow x} H_{f}(a) .
$$

$P$ is a positively homogeneous subadditive functional on $\boldsymbol{C}(\Delta)$, i.e.,

$$
\begin{aligned}
& P\left(f_{1}+f_{2}\right) \leq P\left(f_{1}\right)+P\left(f_{2}\right), \\
& P(k f)=k P(f) \quad \text { for } k \geq 0 .
\end{aligned}
$$

By the Hahn-Banach theorem there exists a linear form $\boldsymbol{F}$ on $\boldsymbol{C}(\Delta)$ such that $F\left(f_{0}\right)=\gamma$ and $F(f) \leq P(f)$ for every $f \in \boldsymbol{C}(\Delta) . \quad F$ is positive, for if $f \leq 0$ then 
$F(f) \leq P(f)=\varlimsup_{a \rightarrow x} H_{f}(a) \leq 0$. Thus $F$ defines a Borel measure $\mu$ on $\Delta$. Further $\mu$ is a probability measure, since $F(1) \leq P(1)=1$ and $F(-1) \leq P(-1)=-1$. To show that $\mu \in \mathscr{M}_{x}$, let $v \in \overline{\mathcal{H}}$ and $v=u+p$, where $u=u_{v}$ is the greatest harmonic minorant of $v$ and $p=p_{v}$ is the potential part of $v$. For $f \in \boldsymbol{C}(\Delta)$ with $f \leq \underline{v}$ (the lower semicontinuous extension of $v$ on $\Delta$ ) we have

$$
H_{f} \leq v=u+p
$$

and then $H_{f} \leq u$. Then

$$
\int f d \mu=F(f) \leq P(f)=\varlimsup_{a \rightarrow x} H_{f}(a) \leq \varlimsup_{a \rightarrow x} u(a)+\lim _{a \rightarrow x} p(a)
$$

and finally

$$
\int \underline{(u+p)} d \mu \leq \bar{u}(x)+\underline{p}(x),
$$

which implies $\mu \in \mathscr{M}_{x}$. On the other hand, since

$$
\int f_{0} d \mu=F\left(f_{0}\right)=\gamma \neq f_{0}(x),
$$

$\mu \neq \varepsilon_{x}$, i.e., $\mathscr{M}_{x} \neq\left\{\varepsilon_{x}\right\}$. Thus the proof is completed.

REMARK. Let $x \in \Delta$ and

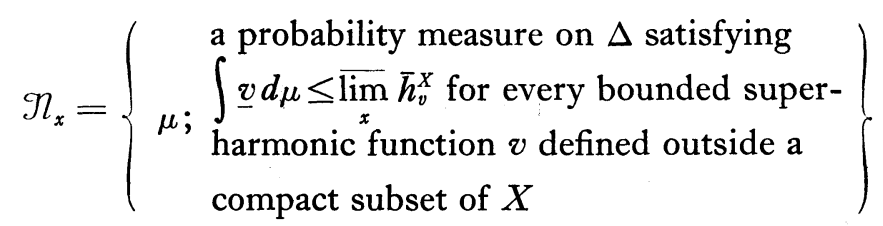

where $\bar{h}_{v}^{X}$ denotes the harmonization of $v^{2}$.

Then, in the same way we can prove that a point $x \in \Gamma$ is regular if and only if $\Re_{x}=\left\{\varepsilon_{x}\right\}$.

Propostion. 1. Let $X^{* *}$ and $X^{*}$ be resolutive compactifications of $X$. And let $X^{*}$ be a quotient space of $X^{* *}$ (i.e., there exists a continuous mapping $\pi$ of $X^{* *}$ onto $X^{*}$ fixing each point of $\left.X\right)$. If $x^{* *} \in \Gamma^{* *}$ is irregular and $\pi^{-1}\left(\pi\left(x^{* *}\right)\right) \cap \Gamma^{* *}$ $=\left\{x^{* *}\right\}$, then $x^{*}=\pi\left(x^{* *}\right)$ is irregular.

In fact, for every measure $\mu^{* *}$ on $\Delta^{* *}$ we define a measure $\mu^{*}$ on $\Delta^{*}$ by

$$
\mu^{*}\left(f^{*}\right)=\mu^{* *}\left(f^{*} \circ \pi\right) \quad \text { for each } f^{*} \in \boldsymbol{C}\left(\Delta^{*}\right) .
$$

From our hypothesis there exists $\mu^{* *} \in \mathscr{M}_{x^{* *}}$ such that $\mu^{* *} \neq \varepsilon_{x^{* *}}$. Since

2) [4], p. 26 . 
$\operatorname{Supp} \mu^{* *} \subset \Gamma^{* *}, \pi\left(y^{* *}\right)=y^{*} \neq x^{*}=\pi\left(x^{* *}\right)$ for every $y^{* *} \in \operatorname{Supp} \mu^{* *} \backslash\left\{x^{* *}\right\}$. We have

$$
\int \underline{v}_{*} d \mu^{*} \leq \int \underline{v}_{* *} d \mu^{* *} \leq \bar{u}_{v}^{* *}\left(x^{* *}\right) \leq \bar{u}_{v}^{*}\left(x^{*}\right)+\underline{p}_{*}\left(x^{*}\right) \quad \text { for every } v \in \overline{\mathcal{H}},
$$

where $\underline{v}_{*}, \underline{v}_{* *}$, etc. denote the lower semicontinuous extensions of $v$ on $\Delta^{*}$, $\Delta^{* *}$, etc.. Thus, we have $\mu^{*} \in \mathscr{M}_{x^{*}}$ with $\mu^{*} \neq \varepsilon_{x^{*}}$.

Before establishing the existence theorem of regular boundary points we shall introduce the family $\mathcal{S}$ of superharmonic functions each of which is bounded from below and is extended finite continuously on $\Gamma$. For $v \in \mathcal{S}$ and $x \in \Gamma$

$$
\varlimsup_{a \rightarrow x} v(a)=\lim _{a \rightarrow x} v(a) .
$$

A non-empty compact subset $E$ of $\Delta$ is termed $T$-extremal if for every $x \in E$ and every $\mu \in \mathscr{M}_{x}$ we have Supp $\mu \subset E$. It is obvious that a family of $T$-extremal sets is inductive and for $x \in \Gamma$, the extremal property of $\{x\}$ implies the regularity of $x$ (Theorem 1 ).

Theorem 2. If for each pair $\left(x_{1}, x_{2}\right)$ of distinct points of $\Gamma$ there exists $v \in \mathcal{S}$ such that $v\left(x_{1}\right) \neq v\left(x_{2}\right)$ then $\Delta$ contains at least one regular point.

Proof. Let $v \in \mathcal{S}$ and $\alpha=\inf \{v(x) ; x \in \Gamma\}$. The number $\alpha$ is finite and $v \geq \alpha$ on $X^{3)}$.

We shall consider

$$
E=\{x \in \Gamma ; v(x)=\alpha\} .
$$

$E$ is a non-empty subset of $\Delta$ and $T$-extremal, since

$$
\int \underline{v} d \mu \leq \vartheta(x)=\underline{v}(x) \quad \text { for every } x \in E \text { and } \mu \in \mathscr{M}_{x} .
$$

By Zorn's lemma, the family of $T$-extremal sets contained in $E$ contains a minimal element $E_{0}$ in the inclusion relation of sets. $E_{0}$ is a non-empty compact subset of $\Gamma$ and $T$-extremal. Suppose for a moment that $E_{0}$ contains two points $x_{1}, x_{2}$. From the hypothesis of the theorem we can find $v_{0} \in \mathcal{S}$ such that $v_{0}\left(x_{1}\right) \neq v_{0}\left(x_{2}\right)$. The set

$$
E_{0}^{\prime}=\left\{x \in E_{0} ; \inf \left\{\underline{v}_{0}(y) ; y \in E_{0}\right\}=\underline{v}_{0}(x)\right\}
$$

is a non-empty compact subset of $E_{0}$ and $E_{0}^{\prime} \neq E_{0}$. If we can show the $T$-extremal property of $E_{0}^{\prime}$, then we are led to a contradiction, since $E_{0}$ is a minimal element, and we can conclude that $E_{0}$ contains only one point $x$, and therefore $x$ is regular. To show that $E_{0}^{\prime}$ is $T$-extremal, suppose that $x \in E_{0}^{\prime}$ and

3) [4], Th. 3.1.6. 
$\mu \in \mathscr{M}_{x}$. Then $x \in E_{0}$ and Supp $\mu \subset E_{0}$. Since

$$
\int \underline{v}_{0} d \mu \leq v_{0}(x)=\underline{v}_{0}(x)
$$

Supp $\mu \subset E_{0}^{\prime}$. Thus the theorem is proved.

Corollary 2. Under the hypothesis of Theorem 2, the lower semicontinuous extension of $v \in \mathcal{S}$ attains its minimum at a regular boundary point.

Theorem 3. If

(1) for every pair $\left(x_{1}, x_{2}\right)$ of distinct points of $\Gamma$ there exists $v \in \mathcal{S}$ so that $v\left(x_{1}\right) \neq v\left(x_{2}\right)$, and

(2) for every $x \in \Gamma$ and for every open neighborhood $U(x)$ of $x$ there exist a neighborhood $V(x)$ of $x$ and a superharmonic function s such that

$$
\left\{\begin{array}{l}
\overline{V(x)} \subset U(x) ; \\
s \text { is bounded from below; } \\
s \text { is extended finite continuously on } \Gamma \cap \overline{V(x)} ; \\
\inf \{\underline{s}(y) ; y \in \Gamma \backslash \overline{V(x)}\}>\inf \{\underline{s}(y) ; y \in \Gamma\}
\end{array}\right.
$$

then $\Gamma$ is the closure of the set of all regular boundary points.

Proof. Let $x \in \Gamma$ and let $U(x), V(x)$ and $s$ be as in (2.5). The set

$$
E=\{x \in \Gamma ; \inf \{\underline{s}(y) ; y \in \Gamma\}=\underline{s}(x)\}
$$

is a non-empty compact subset of $\overline{V(x)}$. As above we can see that $E$ is $T$ extremal and the family of $T$-extremal sets contained in $E$ contains a minimum $E_{0}, E_{0}$ contains only one point $x_{0}$ and $x_{0}$ is regular. Since $x_{0} \in U(x)$, the assertion of Theorem 3 is proved.

In some compactifications we meet the case where the set of all regular boundary points coincides with $\Gamma$. This is, in fact, the case of large compactifications, e.g., the Wiener compactification ${ }^{4}$. Next theorem gives a fairly wide class of such compactifications.

For a family $Q$ of bounded continuous functions on $X$, let $X_{Q}^{*}$ denote the compactification of $X$ such that all functions of $Q$ are extended continuously on $X_{Q}^{*}$ and separate points of $X_{Q}^{*}$.

A resolutive compactification $X^{*}$ of $X$ is termed to be saturated if $X_{Q}^{*}$ is homeomorphic to $X^{*}$, where

$$
Q=\left\{\left.f\right|_{X} ; f \in \boldsymbol{C}\left(X^{*}\right)\right\} \cup\left\{H_{f}: f \in \boldsymbol{C}\left(X^{*} \backslash X\right)\right\}
$$

4) [4], Th. 4.6. 
We know that $X_{Q}^{*}$ is saturated ${ }^{5)}$. In view of the definition of saturated compactification and Lemma 1 we can derive:

Theorem 4. If a resolutive compactification $X^{*}$ of $X$ is saturated, then each point of $\Gamma$ is regular.

\section{Regularity}

In this section, we shall give definitions of regularity strengthened than the usual one and investigate relations among them. The original form of these regularities will be found, for example, in the classical case of Green space [5].

A boundary point $x$ is strongly regular if $x$ has a barrier, i.e., a positive superharmonic function $v$ on $X$ such that $\lim _{a \rightarrow x} v(a)=0$ and inf $\{v(a) ; a \in X \backslash U(x)\}$ $>0$ for every neighbborhood $U(x)$ of $x$.

A boundary point $x$ is called pseudo-strongly regular if for every bounded potential $p$ harmonic in a neighborhood of $x$ we have $\lim _{a \rightarrow x} p(a)=0$.

Proposition 2. The following properties are equivalent:

(1) $x$ is pseudo-strongly regular;

(2) for every bounded and non-negative superharmonic function $v$ on $X$ and for every neighborhood $U(x)$ of $x$ we have

$$
\lim _{a \rightarrow x} R_{v}^{X \backslash \overline{U(x)}}(a)=0 .
$$

Proof. $(1) \Rightarrow(2)$ : let us decompose $R_{v}^{X \backslash \overline{U(x)}}$ into the harmonic part $u$ and the potential part $p$ :

$$
R_{v}^{X \backslash \overline{U(x)}}=u+p .
$$

We remark that both $u$ and $p$ are bounded, and $R_{u}^{X \backslash \overline{U(x)}}=u$ and $R_{p}^{X \mid \overline{U(x)}}=p$. Choose a neighborhood $V(x)$ of $x$ such that $\overline{V(x)} \subset U(x)$. From

$$
\begin{aligned}
& \hat{R}_{R_{u}^{X \backslash \overline{U(x)}}}^{\overline{V(x)} \cap X} R_{u}^{X \backslash \overline{U(x)}} \leq(\sup u) R_{1}^{X \backslash \overline{U(x)}} \\
& \hat{R}_{R_{u}^{X \backslash \overline{U(x)}}}^{\overline{V(x)} \cap X} \leq \hat{R}_{u}^{\overline{V(x)} \cap X} \leq(\sup u) \hat{R}_{1}^{\overline{V(x)} \cap X}
\end{aligned}
$$

we have

$$
\hat{R}_{R_{u}^{X \backslash \overline{U(x)}}}^{V(x) \cap X} \leq(\sup u) \min \left(R_{1}^{X \backslash \overline{U(x)}}, \hat{R}_{1}^{\overline{V(x)} \cap X}\right) .
$$

Since $X^{*}$ is resolutive we see that the right hand side is a potential ${ }^{6)}$.

5) This result can be obtained in the same way as in [3], Prop. 3.2, p. 43.

6) [4], Th. 3.2.23 b). 


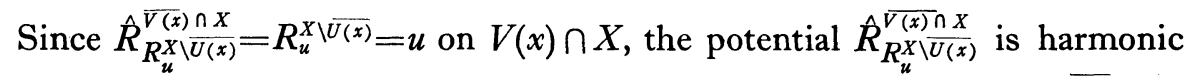
on $V(x) \cap X$, and we have $\lim _{a \rightarrow x} u(a)=0$. We also $\lim _{a \rightarrow x} p(a)=0$, for $R_{p}^{X \backslash \overline{U(x)}}$ is a bounded potential harmonic on $U(x) \cap X$.

$(2) \Rightarrow(1)$ : let $p$ be a bounded potential, which is harmonic on $U_{1}(x) \cap X$ for a neighborhood $U_{1}(x)$ of $x$. We select a neighborhood $U(x)$ of $x$ so that $\overline{U(x)} \subset U_{1}(x)$. Then we have

$$
R_{p}^{X \backslash U(x)}=H_{p}^{U(x) \cap X, X} \quad \text { on } U(x) \cap X^{7)} .
$$

If a superharmonic function $s \geq 0$ satisfies $\lim s \geq p$ on $\partial U=[\overline{U(x)} \backslash U(x)] \cap X$ then $s \geq p$ on $U(x) \cap X{ }^{8)} \quad$ This implies

$$
H_{p}^{U(x) \cap X, X}=p \quad \text { on } U(x) \cap X
$$

and

$$
\varlimsup_{a \rightarrow^{x}} p(a)=\varlimsup_{a \rightarrow x} R_{p}^{X \backslash U(x)}(a)=0, \quad \text { q.e.d. }
$$

Corollary 3. A boundary point $x$ is pseudo-strongly regular if and only if

$$
\lim _{a \rightarrow x} R_{1}^{X \backslash U(x)}(a)=0
$$

for every neighborhood $U(x)$ of $x$.

Proposition 3. A strongly regular boundary point $x$ is pseudo-strongly regular. The converse is true if $X^{*}$ is metrizable.

Proof. Let $p$ be a bounded potential and assume that $p$ is harmonic on $U(x) \cap X$ for a neighborhood $U(x)$ of $x$. By hypothesis, there exists a positive superharmonic function $v$ such that

$$
\lim _{a \rightarrow x} v(a)=0 \quad \text { and } \inf \{v(a) ; a \in X \backslash U(x)\}>\alpha>0 .
$$

Let $A$ be a positive number so that $A \alpha>\sup p$. As in the proof of Proposition 2 , we have

$$
A v \geq p \quad \text { on } U(x) \cap X
$$

which implies $\lim _{a \rightarrow x} p(a)=0$.

Next, suppose $X^{*}$ is metrizable and let $\left\{U_{n}(x)\right\}$ be a base of neighborhoods of $x$ with $\overline{U_{n+1}(x)} \subset U_{n}(x)$. Then

$$
v=\sum_{n=1}^{\infty}\left(1 / 2^{n}\right) R_{1}^{X \backslash \overline{U_{n}(x)}}
$$

7) [4], Cor. 1.2.9.

8) [2], Kor. 2.4.3. 
is a barrier of $x$. In fact, for a positive number $\varepsilon$ we may find an integer $N$ so that

$$
\sum_{n=N+1}^{\infty} 1 / 2^{n}<\varepsilon / 2
$$

Since $x$ is pseudo-strongly regular, by Corollary 3 we can find a neighborhood $W(x)$ of $x$ such that

$$
R_{1}^{X \backslash \overline{U_{n}(x)}}<\varepsilon / 2 \quad \text { on } W(x) \cap X \text { for } n=1,2, \cdots, N .
$$

Then,

$$
v<\varepsilon / 2 \sum_{n=1}^{N} 1 / 2^{n}+\sum_{n=N+1}^{\infty} 1 / 2^{n}<\varepsilon \quad \text { on } W(x) \cap X,
$$

which means $\lim _{a \rightarrow x} v(a)=0$. On the other hand, for an arbitrary $U(x)$ there exists $U_{n}(x)$ with $\overline{U_{n}(x)} \subset U(x)$. Therefore

$$
\begin{aligned}
& \inf \{v(a) ; a \in X \backslash U(x)\} \geq \inf \left\{v(a) ; a \in X \backslash \overline{U_{n}(x)}\right\} \\
& >\inf \left\{\left(1 / 2^{n}\right) R_{1}^{X \backslash \overline{U_{n}(x)}}(a) ; a \in X \backslash \overline{U_{n}(x)}\right\}=1 / 2^{n}>0,
\end{aligned}
$$

Lemma 3. For each point $x \in \Lambda$ there exists a neighborhood $U(x)$ of $x$ such that $R_{1}^{X \backslash U(x)}=1$.

Proof. For a point $x \in \Lambda$ we may find $U(x)$ such that

$$
\overline{U(x)} \cap \Gamma=\phi \text {. }
$$

If a non-negative superharmonic function $s$ satisfies $s \geq 1$ on $X \backslash U(x)$, then $\underline{\lim } s \geq 1$ on $\Gamma$ and $s \geq 1$ on $X$, and finally $R_{1}^{X \backslash U(x)}=1$, q.e.d..

Proposition 4. A pseudo-strongly regular boundary point is regular.

Proof. Let $x$ be pseudo-strongly regular. By Lemma 3, $x \in \Gamma$. We shall show that $\mathscr{M}_{x}=\left\{\varepsilon_{x}\right\}$. Suppose for a moment that there exists $\mu \in \mathscr{M}_{x}$ with $\mu \neq \varepsilon_{x}$. Then,

$$
\mu=\alpha \varepsilon_{x}+\nu, \quad \text { where } 0 \leq \alpha<1 .
$$

We choose a neighborhood $U(x)$ such that

$$
(\operatorname{Supp} \nu) \backslash \overline{U(x)} \neq \phi \text {. }
$$

We then have

$$
\begin{aligned}
\int \underline{\lim } R_{1}^{X \backslash \overline{U(x)}} d \mu & =\alpha \lim _{\overline{a \rightarrow x}} R_{1}^{X \backslash \overline{U(x)}}(a)+\int \lim R_{1}^{X \backslash \overline{U(x)}} d \nu \\
& \leq \varlimsup_{a \rightarrow x} R_{1}^{X \backslash \overline{U(x)}}(a)=0 .
\end{aligned}
$$

On the other hand, the first integral is positive, since 


$$
\int \underline{\lim } R_{1}^{X \backslash \overline{U(x)}} d \nu>0,
$$

which is a contradiction. By Theorem 1, we can prove the proposition.

The following examples (Example 2 and 3) show that the converse of Proposition 4 is not valid in general.

EXAMPLE 1. Let $G$ be a ring domain of the complex plane: $G=\{z ; 1<|z|$ $<4\}$ and $X=G \backslash\{2,3\}$ We consider a usual harmonic structure on $X$, i.e., a function is harmonic if it is continuous and satisfies the Laplace equation. We compactify $X$ in the manner that the boundary consists of two points, one corresponds to $\{z ;|z|=1\} \cup\{2\}$ and the other to $\{z ;|z|=4\} \cup\{3\}$. This is a resolutive compactification, whereas it has no regular boundary point.

Example 2. The one-point compactification of the harmonic space $X$ in Example 1 is resolutive and the boundary point is regular but not pseudostrongly regular.

Example 3. Let $X$ be an open disc in the complex plane endowed with usual topology. For the harmonic structure, we adopt the quotinent sheaf of usual harmonic functions by $k=\operatorname{Re} \frac{1+z}{1-z}$. Consider the closur $\bar{X}$ and identify the point 1 with -1 . This is a compactification of $X$ which is resolutive. In fact, for every continuous function $f$ on $\Delta$, which is a usual continuous function on the boundary circle with $f(1)=f(-1)$, the Dirichlet solution $H_{f}$ is a constant function $f(1)$. Hence the identified point 1 is regular. However, this point is not pseudo-strongly regular, since some neighborhood $U(1)$ is not connected and on a component of $U(1)$ the reduced function of Corollary 3 is constantly 1 .

In view of the above examples, it is natural to ask the conditions under which both regularities coincide. In order to answer the question we consider the following separation condition:

[S] (i) $\Delta$ contains at least two points;

(ii) for every distinct points $x_{1}, x_{2}$ of $\Delta$ (resp. $\left.x_{1} \in X, x_{2} \in \Delta\right)$ there exists $f \in C(\Delta)$ such that

$$
\left.\lim _{\bar{a} \rightarrow x_{1}} H_{f}(a)>\varlimsup_{a \rightarrow x_{2}} H_{f}(a) \quad \text { (resp. } H_{f}\left(x_{1}\right)>\varlimsup_{a \rightarrow x_{2}} H_{f}(a)\right) .
$$

We note that in the inequality in $[S] x_{1}$ and $x_{2}$ are reversible if we take $-f$ instead of $f$.

Theorem 5. Let $X^{*}$ be a resolutive compactification of $X$ satisfying the condition $[S]$. Then the following properties are equivalent: 
(1) $x$ is regular;

(2) $x \in \Gamma$ and for every continuous function $f$ on $\Delta$

$$
\lim _{a \rightarrow x} H_{f}(a) \leq 0 \quad \text { implies } \varlimsup_{a \rightarrow x} H_{f^{+}}(a)=0 ;
$$

(3) $x$ is pseudo-strongly regular.

Proof. Since $(1) \Rightarrow(2)$ and $(3) \Rightarrow(1)$ are immediately seen, it is sufficient to prove $(2) \Rightarrow(3)$. Let $U(x)$ be an open neighborhood of $x$ and $\partial U=[\overline{U(x)} \backslash U(x)]$ $\cap X$. We shall show that for every $y \in \overline{\partial U}$ there exists a non-negative function $f_{y} \in C(\Delta)$ satisfying

$$
\varliminf_{a \rightarrow y} H_{f_{y}}(a)>0=\varlimsup_{a \rightarrow x} H_{f_{y}}(a) .
$$

In fact, by the condition $[S]$, we may find $\varphi \in C(\Delta)$ such that

$$
\lim _{a \rightarrow y} H_{\varphi}(a)>\alpha>\varlimsup_{a \rightarrow x} H_{\varphi}(a) .
$$

Putting $f_{y}=(\varphi-\alpha)^{+}$, we have

$$
\lim _{a \rightarrow y} H_{f_{y}}(a) \geq \lim _{a_{\rightarrow y}} H_{\varphi}(a)-\alpha>0
$$

and

$$
0>\varlimsup_{a \rightarrow x} H_{\varphi}(a)-\alpha \geq \lim _{a \rightarrow x} H_{\varphi-\alpha}(a) .
$$

Therefore, by hypothesis, $\varlimsup_{a \rightarrow x} H_{f y}(a)=0$. Thus, for each point $y \in \overline{\partial U}$ there exists a triple $\left(f_{y}, V(y), \delta_{y}\right)$ such that

$$
\left\{\begin{array}{l}
f_{y} \text { is a non-negative function in } C(\Delta), \\
H_{f y}>\delta_{y} \text { on } V(y) \cap X, \\
\varlimsup_{a \rightarrow x} H_{f_{y}}(a)=0 .
\end{array}\right.
$$

Now, we shall cover $\overline{\partial U}$ by a finite system $\left\{V\left(y_{i}\right)\right\}_{i=1}^{n}$ of such $V(y)$, and let $\delta=\min \left\{\delta_{y_{i}} ; 1 \leq i \leq n\right\}, f=\sum_{i=1}^{n} f_{y_{i}}$ and $V=\bigcup_{i=1}^{n} V\left(y_{i}\right)$. It is easily seen that

$$
H_{f} \geq \delta \text { on } V \cap X \text { and } \lim _{a \rightarrow x} H_{f}(a)=0 .
$$

We may find a non-negative superharmonic function $s_{0}$ such that ${ }^{9)}$

$$
\varlimsup\left(H_{1}^{U(x) \cap X, X}-\varepsilon s_{0}\right) \leq \begin{cases}1 & \text { on } \partial U \\ 0 & \text { on } \overline{U(x)} \cap \Delta\end{cases}
$$

for every $\varepsilon>0$.

9) [4], Th. 1.2.3. 
In view of this, it is derived that

$$
\underline{\lim }\left[1 / \delta H_{f}-\left(H_{1}^{U(x) \cap X, X}-\varepsilon s_{0}\right)\right] \geq 0 \text { on } \partial U \cup(\overline{U(x)} \cap \Delta) .
$$

Hence $1 / \delta H_{f} \geq H_{1}^{U(x) \cap X, X}-\varepsilon s_{0}$, and $\varepsilon$ being arbitrary, $1 / \delta H_{f} \geq H_{1}^{U(x) \cap X, X}=$ $R_{1}^{X \backslash U(x)}$ on $U(x) \cap X$, which implies $\lim _{a \rightarrow x} R_{1}^{X \backslash U(x)}(a)=0$. Thus, by Corollary $3, x$ is pseudo-strongly regular, q.e.d..

In the same way we obtain:

Theorem 6. Suppose that $x$ is regular and following condition is fulfilled: $[T]$ for every point $y \in X^{*}$ distinct from $x$ there exists a non-negative superharmonic function $v$ such that

$$
\lim _{a \rightarrow y} v(a)=0<\lim _{a \rightarrow x} v(a) .
$$

Then $x$ is pseudo-strongly regular.

We can see later $(\$ 5)$ that when we consider a relatively compact open set as a harmonic space and its closure as a compactification, the condition $[T]$ is fulfilled. Thus all regular boundary points of relatively compact open sets are pseudo-strongly regular and therefore strongly regular.

REMARK. If we drop boundedness from the definition of pseudo-strong regularity we shall be led to an exceedingly strong condition. For, if we have $\lim _{a \rightarrow x} p(a)=0$ for every potential $p$ which is harmonic in a neighborhood of $x$, then $x$ is completely regular, i.e., $\lim _{a \rightarrow x} H_{f}(a)=f(x)$ for every resoluvtive (not necessarily bounded) function $f$ continuous at $x$.

At the end of this section, we give a condition which affirms a boundary point to be regular.

Theorem 7. Let $\mathcal{U}(x)$ be a fundamental system of neighborhoods of $x$. If $\lim _{\overline{U(x)}}\left[\varlimsup_{a \rightarrow x} R_{1}^{X \backslash U(x)}(a)\right]<1$, then $x$ is regular.

Proof. First, we shall show that $x \in \Gamma$. For, otherwise by Lemma 3 there would exist $U(x) \in \mathcal{U}(x)$ such that $R_{1}^{X \backslash U(x)}=1$.

Let $\alpha_{0}=\lim _{\overline{U(x)}}\left[\varlimsup \lim _{1}^{X \backslash U(x)}(a)\right]$. By hypothesis, for every neighborhood $V(x)$ of $x$ there exists $U_{1}(x) \in \mathcal{U}(x)$ such that

$$
U_{1}(x) \subset V(x) \text { and } \varlimsup_{a \rightarrow x} R_{1}^{X \backslash U_{1}(x)}(a)<1 .
$$

Hence, we find $W_{1}(x) \in \mathcal{U}(x)$ and $\alpha_{1}$ satisfying 


$$
\left\{\begin{array}{l}
W_{1}(x) \subset U_{1}(x), \\
\alpha_{0}<\alpha_{1}<1, \\
R_{1}^{X \backslash U_{1}(x)} \leq \alpha_{1} \text { on } W_{1}(x) \cap X .
\end{array}\right.
$$

Therefore, for every neighborhood $V(x)$ of $x$ there exist $V_{1}(x), U_{1}(x), W_{1}(x) \in$ $\mathcal{U}(x)$ and $v_{1}=R_{1}^{X \backslash U_{1}(x)}$ such that

$$
\left\{\begin{array}{l}
W_{1}(x) \subset U_{1}(x) \subset V_{1}(x) \subset \overline{V_{1}(x)} \subset V(x), \\
v_{1} \leq \alpha_{1} \text { on } W_{1}(x) \cap X, \\
v_{1}=1 \text { on } X \backslash V(x) .
\end{array}\right.
$$

Next, for $V_{2}(x) \in \mathcal{U}(x)$ with $\overline{V_{2}(x)} \subset W_{1}(x)$ one may find $U_{2}(x) W_{2}(x) \in \mathcal{U}(x)$ satisfying

$$
\begin{aligned}
& W_{2}(x) \subset U_{2}(x) \subset V_{2}(x), \\
& R_{1}^{X \backslash U_{2}(x)} \leq \alpha_{1} \text { on } W_{2}(x) \cap X .
\end{aligned}
$$

Since $R_{v_{1} / \alpha_{1}}^{X \backslash U_{2}(x)} \leq R_{1}^{X \backslash U_{2}(x)}$ on $U_{2}(x) \cap X$, if we put $v_{2}=\hat{R}_{v_{1}}^{X \backslash U_{2}(x)}$, then we have

$$
\left\{\begin{array}{l}
v_{2} \leq \alpha_{1}^{2} \text { on } W_{2}(x) \cap X \\
v_{2}=1 \text { on } X \backslash V(x) .
\end{array}\right.
$$

By induction, we can construct $U_{n}(x), W_{n}(x) \in \mathcal{U}(x)$ and $v_{n}=\hat{R}_{v_{n-1}}^{X \backslash U_{n}(x)}$ such that

$$
\left\{\begin{array}{l}
W_{n}(x) \subset U_{n}(x) \subset V_{n}(x), \\
v_{n} \leq \alpha_{1}^{n} \text { on } W_{n}(x) \cap X, \\
v_{n}=1 \text { on } X \backslash V(x) .
\end{array}\right.
$$

To prove Theorem it is sufficient to show that $\mathscr{M}_{x}=\left\{\varepsilon_{x}\right\}$. Suppose, for a moment, that $\mathscr{M}_{x}$ contains $\mu$ such that $\mu=a \varepsilon_{x}+\nu$ and $0 \leq a<1$. We may find $V_{0}(x) \in \mathcal{U}(x)$ with the property $\nu\left(\Delta \backslash V_{0}(x)\right)>(1-a) / 2$. If we construct above $V_{n}(x), U_{n}(x), W_{n}(x)$ and $v_{n}$ starting from $V(x)$ with $\overline{V(x)} \subset V_{0}(x)$, then we are led to a contradiction. For, choosing $n$ so large that $(1-a) / 2>\alpha_{1}^{n}$ we have

$$
\alpha_{1}^{n} \geq \varlimsup_{a \rightarrow x} v_{n}(a) \geq \int \underline{v}_{n} d \mu \geq \int \underline{v}_{n} d \nu \geq \nu\left(\Delta \backslash V_{0}(x)\right)>(1-a) / 2 .
$$

Thus the proof is completed.

\section{Characterization of the harmonic boundary}

In this section, we shall give a characterization of the harmonic boundary $\Gamma$ as a minimal determining set for some function families, i.e., the Silov boundary. As a consequence one can derive a condition under which $\Gamma$ is the closure of the set of all regular points. First we prove: 
Theorem 8. Let $X^{*}$ be a resolutive compactification of $X$. Then

$$
\Gamma=\left\{x \in \Delta ; \lim _{a \rightarrow x} R_{1}^{X \backslash U(x)}(a)=0 \text { for every neighborhood } U(x)\right\}
$$

and

$$
\Gamma=\left\{x \in \Delta ; R_{1}^{X \backslash U(x)} \neq 1 \text { for every neighborhood } U(x)\right\} .
$$

Proof. Let $A$ and $B$ be the sets described on the right hand of (4.1) and (4.2), respectively. It is obvious that $A \subset B$, and by Lemma $3, B \subset \Gamma$ is derived immediately. In order to complete the proof, we shall show that $\Gamma \subset A$. Let $x \in \Gamma$ and, for a moment, suppose that $x \notin A$. Then one can find an open neighborhood $U(x)$ of $x$ and a number $\alpha$ such that

$$
0<\alpha<1 \text { and } R_{1}^{X \backslash U(x)} \geq \alpha \text { on } U(x) \cap X .
$$

For an open neighborhood $V(x)$ of $x$ with $\overline{V(x)} \subset U(x), p=\min \left(\hat{R}_{1}^{X \backslash U(x)}, \hat{R}_{1}^{V(x) \cap X}\right)$ is a potential. Since $p=\hat{R}_{1}^{X \backslash U(x)}$ on $V(x) \cap X, p \geq \alpha$ on the same set, which contradicts $x \in \Gamma$, q.e.d..

To proceed the minimal property of the harmonic boundary, we recall that

$$
\overline{\mathcal{H}}=\{v: v \text { is a superharmonic function, bounded from below }\} \text {. }
$$

Further we define

$$
\overline{\mathcal{H}}^{\prime}=\{u+p ; u \text { is a bounded harmonic function and } p \text { is a potential }\} \text {. }
$$

Of course, $\overline{\mathcal{H}^{\prime}}$ contains all bounded harmonic functions and is contained in $\overline{\mathcal{H}}$.

Theorem 9. $\Gamma$ is the $\overline{\mathcal{H}}$ (resp. $\left.\overline{\mathcal{H}^{\prime}}\right)$-Šilov boundary.

Proof. We know that $\Gamma$ is $\overline{\mathcal{H}}$-determinig ${ }^{10)}$. Let $S$ be a non-empty compact subset of $\Delta$, determining for $\overline{\mathcal{H}}$ (resp. $\overline{\mathcal{H}^{\prime}}$ ). We shall prove that $\Gamma \subset S$. Suppose, for a moment, that $x \in \Gamma \backslash S$. Then for every point $x^{\prime} \in S$ there exist $f_{x^{\prime}} \in \boldsymbol{C}(\Delta)$ and a potential $p_{x^{\prime}}$ such that

$$
\left\{\begin{array}{l}
f_{x^{\prime}} \geq 0, \quad f_{x^{\prime}}(x)=0, \\
\lim _{a \rightarrow x^{\prime}}\left(H_{f_{x^{\prime}}}(a)+p_{x^{\prime}}(a)\right)>\lim _{\overline{a \rightarrow x}} H_{f_{x^{\prime}}}(a)=0 .
\end{array}\right.
$$

In fact, (1) for $x^{\prime} \in \Gamma$, we may choose $f_{x^{\prime}}$ and $p_{x^{\prime}}$ so that $f_{x^{\prime}}\left(x^{\prime}\right)>f_{x^{\prime}}(x)=0$ and $\underline{\lim }\left(H_{f_{x^{\prime}}}+p_{x^{\prime}}\right) \geq f_{x^{\prime}}$ on $\Delta$, and (2) for $x^{\prime} \in \Lambda$ we may only choose $f_{x^{\prime}}=0$ and $p_{x^{\prime}}$, satisfying $\lim _{a \rightarrow x^{\prime}} p_{x^{\prime}}(a)=+\infty^{11)}$.

In view of (4.4), one may find a neighborhood $V\left(x^{\prime}\right)$ of $x^{\prime}$ and a positive number $\delta_{x^{\prime}}$ such that

10) [4], Th. 3.1.5.

11) [4], Th. 3.1.3. 


$$
H_{f_{x^{\prime}}}+p_{x^{\prime}} \geq \delta_{x^{\prime}} \quad \text { on } V\left(x^{\prime}\right) \cap X .
$$

A finite number of $V\left(x^{\prime}\right)$, say $\left\{V\left(x_{i}^{\prime}\right)\right\}_{i=1}^{n}$, covers $S$. Letting $f=\sum_{i=1}^{n} f_{x_{i}^{\prime}}$, $\delta=\min \left\{\delta_{x_{i}} ; 1 \leq i \leq n\right\}$ and $p=\sum_{i=1}^{n} p_{x_{i}}$, we conclude that

$$
\underline{\left(H_{f}+p\right)} \geq \delta \quad \text { on } S,
$$

and $H_{f}+p \geq \delta$, since $S$ is $\overline{\mathscr{H}}$ (resp. $\overline{\mathcal{H}^{\prime}}$ )-determining. This implies also $H_{f} \geq \delta$. On the other hand, by Lemma $1, \delta \leq \lim _{a \rightarrow x} H_{f}(a) \leq f(x)=0$, which is a contradiction. Thus $\Gamma \subset S$, q.e.d..

Let $\mathscr{F}$ be a family of bounded harmonic functions containing $\left\{H_{f} ; f \in\right.$ $\boldsymbol{C}(\Delta)\}$. We assume that a compactification satisfies the following condition $\left[S^{\prime}\right]$ weaker than $[S]$ in $\S 3$.

$\left[S^{\prime}\right]$ for every distinct $x_{1}, x_{2} \in \Delta$ there exists a function $f \in C(\Delta)$ such that $\lim _{a \rightarrow x_{1}} H_{f}(a)>\varlimsup_{a \rightarrow x_{2}} H_{f}(a)$.

We have then

Theorem 10. Suppose that the condition $\left[S^{\prime}\right]$ is fulfilled. Let $R$ be the set of all regular boundary points. Then, $R$ is dense in $\Gamma$ if and only if $\bar{R}$ is $\mathscr{F}$-determining.

Proof. It will be sufficient to show that $\Gamma$ is the $\mathscr{F}$-Silov boundary. Let $S$ be a compact subset of $\Delta$ which is $\mathscr{F}$-determining and suppose that $x \in \Gamma \backslash S$. By the condition $\left[S^{\prime}\right]$, for every $y \in S$ we may find $f_{y} \in \boldsymbol{C}(\Delta)$ such that

$$
\lim _{a \rightarrow y} H_{f_{y}}(a)>0=\varlimsup_{a \rightarrow x} H_{f_{y}}(a) \geq f_{y}(x)
$$

and consequently there exists a neighborhood $U(y)$ of $y$ and a positive number $\delta_{y}$ satisfying

$$
H_{f_{y}}>\delta_{y} \quad \text { on } U(y) \cap X .
$$

A finite number of such $U(y)$, say $\left\{U\left(y_{i}\right)\right\}_{i=1}^{N}$, covers $S$. Putting $f=\max \left\{f_{y_{i}}\right.$; $1 \leqq i \leqq N\}$ and $\delta=\min \left\{\delta_{y_{i}} ; 1 \leqq i \leqq N\right\}$, we have

$$
\lim H_{f} \geq \delta>0 \quad \text { on } S \text {. }
$$

Since $S$ is $\mathscr{F}$-determining, $H_{f} \geq \delta$ on $X$, but this leads to a contradiction

$$
0 \geq f(x) \geq \lim _{a \rightarrow x} H_{f}(a) .
$$

Thus we have proved that every $\mathscr{F}$-determining set contains $\Gamma$. Since $\Gamma$ itself is $\mathscr{F}$-determining, it is the $\mathscr{T}$-Šsilov boundary. 


\section{Regular boundary points of open subsets}

In the present section we consider an open subset of $X$ as a harmonic space and discuss the regular boundary points for the Dirichlet problem.

Let $G$ be an open subset of $X$. We may introduce into $G$ a harmonic structure from that of $X$. We may compactify $G$ so that its compactification $G^{\Omega}$ contains the boundary $\partial G \cup\{\omega\}$, where $\partial G$ is a relative boundary and $\omega$ is an ideal boundary. In the compactification $G^{\Omega}$ deleted neighborhood of $\omega$ is the intersection of $\partial G \cup G$ with the complement of a compact subset of $X$. We consider the Dirichlet problem on $G^{\mathrm{Q}}$. Dirichlet solutions for functions which vanish at $\omega$, i.e., normalized solutions are frequently used. To construct normalized solutions precisely, let $f$ be a function on $G^{\Omega} \backslash G$ and we consider

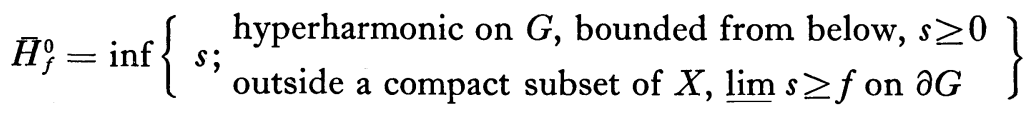

and $\underline{H}_{f}^{0}=-\bar{H}_{(-f)}^{0}$. If $\bar{H}_{f}^{0}=\underline{H}_{f}^{0}$ and harmonic, this is called a normalized solution and is denoted by $H_{f}^{0}$. It is known that every bounded Baire function has a normalized solution. ${ }^{12)}$

It is immediately seen that for a bounded Baire function $f$ on $G^{\mathbf{Q}} \backslash G$ vanishing at $\omega$, we have $H_{f}^{0}=H_{f}$, where $H_{f}$ is the solution considered in the preceding sections. We shall show

Proposition 5. $G^{\mathrm{Q}}$ is a resolutive compactification.

Proof. Let $f \in \boldsymbol{C}\left(G^{\alpha}\right), f(\omega)=a$ and

$$
\bar{h}_{f}^{G, X}=\inf \left\{\begin{array}{c}
\text { hyperharmonic on } G, \text { possessing non-positive } \\
s ; \text { subharmonic minorant, } s \geq f \text { outside a compact } \\
\text { subset of } X, \underline{\lim } s \geq 0 \text { on } \partial G
\end{array}\right\}
$$

and $\underline{h}_{f}^{G, X}=-\bar{h}_{(-f)}^{G, X}$.

The following inequlities are derived immediately from the definition

$$
\bar{H}_{\mathrm{x}_{\{\omega\}}} \leq \bar{h}_{1}^{G, X} \text { and } \quad \underline{H}_{\mathrm{x}_{\{\omega\}}} \geq \underline{h}_{1}^{G, X},
$$

where $\chi_{\{\omega\}}$ is the characteristic function of $\{\omega\}$. Since the constant function 1 is harmonic, $\bar{h}_{1}^{G, X}=\underline{h}_{1}^{G, X}{ }^{13)}$. We have thus

$$
a H_{\chi_{\{\omega\}}}=h_{a}^{G, X} .
$$

Denoting by

12) [4], Th. 1.2.7.

13) [4], Cor. 2.2.3. 


$$
f_{1}= \begin{cases}f & \text { on } \partial G \\ 0 & \text { at } \omega\end{cases}
$$

and $f_{2}=a \chi_{(\omega)}, f=f_{1}+f_{2}$. Hence

$$
\underline{H}_{f_{1}}+H_{f_{2}} \leq \underline{H}_{f} \leq \bar{H}_{f} \leq \bar{H}_{f_{1}}+H_{f_{2}} .
$$

But, by what we have remarked above $\underline{H}_{f_{1}}=\bar{H}_{f_{1}}$ since $f_{1}$ is a bounded Baire function vanishing at $\omega$, q.e.d..

We shall note that if a point $x \in \partial G$ is regular, then for every resolutive function $f$ which is bounded and continuous at $x$ we have

$$
\lim _{a \rightarrow x} H_{f}(a)=f(x) .
$$

Next, we shall show that every regular boundary point of an open subset $G$ of $X$ is pseudo-strongly regular. If we prove the following proposition, this will be seen immediately by the argument used in the proof of Theorem 5 . (Cf. also Theorem 6).

Proposition 6. Let $x \in \partial G$ be regular and let $U(x)$ be a relatively compact neighborhood of $x$ and $K=\partial U(x) \cap \bar{G}$. For every $y \in K$ there exists a non-negative superharmonic function $v$ in $G$ satisfying

$$
\lim _{a \rightarrow x} v(a)=0<\lim _{\overline{a \rightarrow y}} v(a) .
$$

Proof. Let $p_{0}$ be a positive continuous potential on $X$ with $p_{0}(x) \neq p_{0}(y)^{14)}$. In the case (i) $p_{0}(x)<p_{0}(y), v=H_{\max }^{0}\left(p_{0}-p_{0}(x), 0\right)+\left[p_{0}-H_{p_{0}}^{0}\right]$ is the desired one, where $H^{0}$ denotes the normalized solution in $G$. Indeed, $\lim _{a \rightarrow x} H_{f}^{0}(a)=f(x)$ for every Baire function continuous at $x$ implies $\lim _{a \rightarrow x} v(a)=0$, and $\lim _{\bar{a} \rightarrow y} v(a) \geq$ $\lim _{a \rightarrow y}\left[H_{p_{0}-p_{0}(x)}^{0}(a)+p_{0}(a)-H_{p_{0}}^{0}(a)\right] \geq-p_{0}(x)+p_{0}(y)>0$. In the case (ii) $p_{0}(x)>$ $p_{0}(y), v=H_{f}$ fulfills the requirement, where

$$
f= \begin{cases}\max \left(p_{0}(x)-p_{0}, 0\right) & \text { on } \partial G \\ p_{0}(x) & \text { at } \omega .\end{cases}
$$

For, putting

$$
f_{1}= \begin{cases}\max \left(p_{0}(x)-p_{0}, 0\right) & \text { on } \partial G \\ 0 & \text { at } \omega\end{cases}
$$

and

$$
f_{2}= \begin{cases}0 & \text { on } \partial G \\ p_{0}(x) & \text { at } \omega\end{cases}
$$

14) [2], Kor. 2.7.3. 
we have $f=f_{1}+f_{2}$ and $H_{f}=H_{f_{1}}+h_{p_{0}(x)}^{G, x}$. Thus we have $\lim _{a \rightarrow x} v(a)=0$ since $h_{p_{0}(x)}^{G \cdot X}=p_{0}(x) H_{x_{(\omega)}}$. If we put

$$
g_{1}= \begin{cases}\max \left(-p_{0},-p_{0}(x)\right) & \text { on } \partial G \\ 0 & \text { at } \omega\end{cases}
$$

and

$$
g_{2}=p_{0}(x) \text { (the constant function), }
$$

then $g_{1}$ and $g_{2}$ are resolutive and $f=g_{1}+g_{2}$. We have hence

$$
\begin{aligned}
\lim _{a \rightarrow y} v(a) & =\lim _{a \rightarrow y}\left[H_{g_{1}}(a)+p_{0}(x)\right] \geq \lim _{a \rightarrow y}\left(H_{-p_{0}}^{0}(a)+p_{0}(x)\right) \\
& \geq \lim _{a \rightarrow y}\left(p_{0}(x)-p_{0}(a)\right)>0, \text { q.e.d. }
\end{aligned}
$$

In view of this proposition we have

Theorem 11. Let $G$ be an open subset of $X$. Every regular boundary point is strongly regular and, in particular, regularity of boundary points is a local property.

\section{Osaka City University}

\section{References}

[1] H. Bauer: Šilovscher Rand und Dirichletsches Problem, Ann. Inst. Fourier 11 (1961) 89-136.

[2] H. Bauer: Harmonische Räume und ihre Potentialtheorie (Lecture Notes in Math. 22) Berlin-Heidelberg-New York, Springer 1966.

[3] T. Ikegami: A note on axiomatic Dirichlet problem, Osaka J. Math. 6 (1969), 39-47.

[4] C. Meghea: Compactifications des espaces harmoniques (Lecture Notes in Math. 222) Berlin-Heidelberg-New York, Springer 1971.

[5] L. Naïm: Sur le rôle de la frontière de R.S. Martin dans la théorie du potentiel, Ann. Inst. Fourier 7 (1957) 183-281. 
